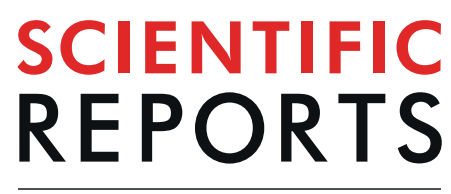

natureresearch

\title{
OPEN CD138 plasma cells may predict brain metastasis recurrence following resection and stereotactic radiosurgery
}

Received: 29 January 2019

Accepted: 14 August 2019

Published online: 07 October 2019

\author{
Michael H. Soike ${ }^{1}$, Jennifer Logue ${ }^{2}$, Shadi Qasem ${ }^{3}$, Ryan T. Hughes $\mathbb{1}^{4}{ }^{4}$ Emory McTyre ${ }^{5}$, \\ Jing Su ${ }^{6}$, Pierre Triozzi ${ }^{7}$, Maurizio Bendandi ${ }^{7}$, Hui-Wen Lo $^{8}$, Tamjeed Ahmed ${ }^{7}$, \\ Stacey S. O'Neill ${ }^{9}$, Waldemar Debinski ${ }^{8}$, Boris Pasche $\mathbb{1}^{7}$, Kounosuke Watabe ${ }^{8}$, \\ Lance D. Miller ${ }^{8}$, Michael D. Chan ${ }^{4}$ \& Jimmy Ruiz ${ }^{7,10}$
}

We sought to identify candidate biomarkers for early brain metastasis (BM) recurrence in patients who underwent craniotomy followed by adjuvant stereotactic radiosurgery. RNA sequencing was performed on eight resected brain metastasis tissue samples and revealed B-cell related genes to be highly expressed in patients who did not experience a distant brain failure and had prolonged overall survival. To translate the findings from RNA sequencing data, we performed immunohistochemistry to stain for $B$ and $T$ cell markers from formalin-fixed parffin-embedded tissue blocks on 13 patients. CD138 expressing plasma cells were identified and quantitatively assessed for each tumor sample. Patients' tumor tissues that expressed high levels of CD138 plasma cells $(N=4)$ had a statistically significant improvement in OS compared to low levels of $C D 138(N=9)(p=0.01)$. Although these findings are preliminary, the significance of $C D 138$ expressing plasma cells within BM specimens should be investigated in a larger cohort. Immunologic markers based on resection cavity analysis could be predictive for determining patient outcomes following cavity-directed SRS.

Approximately 170,000 patients in the United States are diagnosed with brain metastasis (BM) every year ${ }^{1}$. Prognosis can vary considerably depending on factors such as the primary site of disease, histology, burden of systemic disease, and intracranial disease ${ }^{2-6}$. A standard treatment option for limited brain metastases from solid tumor primaries is stereotactic radiosurgery (SRS), which allows for rapid treatment of brain metastases with preservation of cognition, but it is an expensive modality compared to its alternative, whole brain radiation therapy (WBRT) and often requires a specialized center ${ }^{7-10}$.

One limitation of SRS is the potential need and expense of additional treatments for distant brain failure $(\mathrm{DBF})^{11,12}$. Methods of scoring clinical and histopathologic factors have attempted to predict intracranial failure, but these have been met with modest successes, predominantly because of the heterogeneity of the brain metastasis population ${ }^{13-15}$. Developing an accurate biomarker that can help predict for improved DBF or OS in patients with brain metastases treated with SRS would be a valuable tool.

Specific patterns of immune cells within the tumor microenvironment are associated with improved outcome in patients with many types of cancers, regardless of the type of therapy administered ${ }^{16}$.

\footnotetext{
${ }^{1}$ Hazlerig-Salter Radiation Oncology Center, University of Alabama at Birmingham, Birmingham, AL, 35249, USA. ${ }^{2}$ University of South Florida Morsani College of Medicine at H. Lee Moffitt Cancer Center, 12902, Tampa, FL, 33612, USA. ${ }^{3}$ Department of Pathology and Laboratory Medicine, University of Kentucky School of Medicine, Lexington, KY, USA. 'Department of Radiation Oncology, Wake Forest School of Medicine, Winston-Salem, NC, 27157, USA. ${ }^{5}$ Department of Radiation Oncology, Greenville Health System Cancer Institute, Greenville, SC, 29605, USA. ' Department of Biostatistical Sciences, Wake Forest School of Medicine, Winston-Salem, NC, 27157, USA. ${ }^{7}$ Department of Medicine (Hematology \& Oncology), Wake Forest School of Medicine, Winston-Salem, NC, 27157, USA. ${ }^{8}$ Department of Cancer Biology, Wake Forest School of Medicine, Winston-Salem, NC, 27157, USA. ${ }^{9}$ Department of Pathology, Wake Forest School of Medicine, Winston-Salem, NC, 27157, USA. ${ }^{10}$ W.G. (Bill) Hefner Veteran Administration Medical Center, Cancer Center, Salisbury, NC, United States of America. Correspondence and requests for materials should be addressed to M.H.S. (email: msoike@gmail.com)
} 
Tumor infiltrating lymphocytes (TILs) of variable density can be observed in brain metastases and are typically composed of various cell types with a higher fraction of T cells than B cells. The presence of TILs, CD3 and CD8 T cells in specific, has been associated with improved survival as compared to patients with only sparse or scattered TIL infiltration ${ }^{17}$. Furthermore, the Immunoscore, which is based on an automated calculation of the $\mathrm{CD} 3 / \mathrm{CD} 8$ ratio among TILs, has been shown to have independent prognostic significance in patients with brain metastases ${ }^{18}$. How TILs influence the response of brain metastasis to SRS is not known.

To identify potential immune biomarkers that could be predictive of outcomes in brain metastasis patients, we performed a retrospective study and tissue analysis of patients who have undergone surgical resection of a brain metastasis followed by SRS. In the initial group of patients, we used microarray to identify immune signatures from resected brain metastasis tissue. We then expanded this microarray profile and sought to characterize immune cells within the brain metastasis tissue with immunohistochemistry associated with favorable outcomes.

\section{Methods}

Patient selection. This study was approved by the Wake Forest Institutional Review Board IRB00008427. Patients signed informed consent for advanced tissue tumor banking prior to analysis. We selected 8 patients with new brain metastases from various primary tumors who underwent craniotomy and adjuvant SRS. These samples were frozen brain metastasis tissues were collected from patients who consented to the Wake Forest Brain Tumor Center of Excellence Tumor Tissue Bank. These samples were analyzed with RNA sequencing. Six of the original 8 patients had their formalin-fixed paraffin-embedded (FFPE) tissue also evaluated by immunohistochemistry (IHC) and 7 additional patients with available FFPE tissues who had craniotomy and received adjuvant SRS were also included in the study. All methods were performed in accordance with institutional policies, particularly for genomic analysis. A Clinical Laboratory Improvement Amendments certified laboratory was utilized for immunohistochemistry analysis of FFPE tissue with a board certified pathologist (SQ) reviewing the slides.

Stereotactic radiosurgery. SRS was performed on the Leksell Gamma Knife Model C (Elekta, Stockholm, Sweden) prior to May 2009, and Perfexion after May 2009. Same day headframe fixation was used for immobilization. Patients underwent a contrast-enhanced stereotactic magnetic resonance imaging scan of the brain with headframe in place. The GammaPlan Treatment Planning System (AB Elekta, Stockholm, Sweden) was used to develop the treatment plan. Median marginal dose prescribed was 18-22 Gy and was generally prescribed to the $50 \%$ isodose line. The dose selected was based on guidelines previously described by Shaw et al. ${ }^{19}$. The targeting of the resection cavity of the metastasis was previously described by Jensen et $_{\text {al }}{ }^{20}$.

Patient follow-up. After adjuvant SRS, patients were followed with repeat MRI approximately 1-2 months later, and then every 3 month basis for 2 years. Distant brain failures (DBF) were determined to be new metastases that developed outside of the prior SRS volume.

RNA sequencing. We identified eight samples from patients who had craniotomy for a new diagnosis of brain metastasis. The frozen brain tumor tissue was assessed using standard sectioning and evaluation for tumor content and viability by a board certified pathologist. Areas with adequate cellularity and viability were selected for testing. Total RNA was purified from the frozen specimens using the RNeasy Plus Micro Kit (Qiagen) with genomic DNA removal. RNA integrity (RIN) was determined by electrophoretic tracing using an Agilent Bioanalyzer. RNAseq libraries were constructed from samples (RIN > 7.0) using the Illumina TruSeq Stranded Total RNA kit with Ribo-Zero rRNA depletion. Indexed libraries were sequenced on an Illumina NextSeq 500 DNA sequencer using $150 \times 150$-nt paired end reads, generating $>40$ million reads per sample $(12$ samples per flow cell) with $>80 \%$ of sequences achieving $>$ Q30 Phred quality scores. Quality of raw sequencing reads were assessed by FASTQC analysis (Babraham Bioinformatics). Sequence reads were aligned using the STAR sequence aligner $^{21}$, and gene counts determined using featureCounts software ${ }^{22}$. Differential gene expression was analyzed using the DESeq2 algorithm ${ }^{23}$. Significant genes were defined as $\mathrm{p}<0.05$ after adjustment for false discovery (Benjamini-Hochberg). Genes and samples were hierarchically clustered using Pearson correlation as the distance metric and visualized by heatmap analysis (Fig. 1).

Immunohistochemistry (IHC). After initial RNA sequencing was performed in a CLIA certified laboratory, IHC was performed on a larger cohort of patients for proteins of interest, 13 in total, including 6 of the previous patients who underwent RNA Seq to correlate the findings from RNA sequencing. Two of the patients in the initial genomic sampling were excluded from IHC analysis because these patients did not meet the criteria of craniotomy followed by adjuvant SRS.

Cases with corresponding formalin-fixed paraffin-embedded tissue were selected for immunohistochemical testing. IHC stains were performed according to validated procedures using DAKO Link 48 autostainer (Agilent technologies, Santa Clara, CA, USA). The tissue was stained for CD20 (DAKO), CD3 (DAKO), CD138-expressing plasma cells (DAKO), Programmed cell death protein-1 (PD1) (Spring Bioscience) and Program death ligand-1 (PDL-1) (Spring Bioscience). Immunohistochemistry stains for T and B cell lymphocyte markers were chosen due to a genomic analysis of a smaller group of heterogeneous patients that revealed high expression of lymphocyte genomic products. The stains were scored on a scale of $0-3$ by a board-certified pathologist who was blinded to the immune signature profiles. The number of cells expressing CD138 was quantitatively assessed per 40x high power field. The difference in assessment methods was due to the pattern of distribution of CD138, which had patchy infiltration whereas the other markers showed diffuse infiltration. 

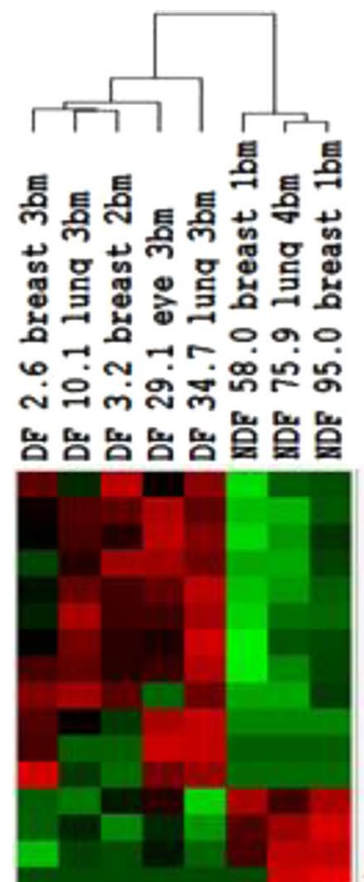

UTRN | ENSG00000152818.14 | 4241.4

ARMCX1 I ENSG00000126947.7 | 267.

ADAM33 I ENSGOODO0149451,13 | 23.4

BTBD7P1 | ENSG00000203414.2 | 10.C

SOS2 I ENSG00000100485.7 i 1243.7] C14orf159 | BNSG00000133943.16 |: LPL | ENSGO0000175445.10 | 757.971 MIR4458HG I ENSG00000247516.3 I 16 MUC3A I ENSG00000169894.13 | 41.31 HOXD3 | ENSG00000128652.7 | 61.88: HOXD4 ENSG00000170166.5 | 21

RP11-8L2.1 | ENSG00000250546.1 | ] ALDOC I ENSG00000109107.9 | 1118.: ZSCAN29 | ENSGO0000140265.8 | 631. SPATA5L1 | EASG00000171763.13 | 18 * IGHV4-28 ENSG00000211952.2 7.2 TRIM31 | ENSG00000204616.6 | 7.828 MDGA1 | ENSG00000112139.10 | 159.5 PEX6 | ENSG00000124587.9 | 463.571 TFF1 ENSG00000160182.2 712.371 PXDNL I ENSG00000147485.8 | $34.82 \varepsilon$ SLC1A5 I EASG00000105281.8 | 2793. * IGHV1-24 | ENSG00000211950.2 | 16. ZSCAN1 I RASG00000152467.5 | 14.08 F2RL2 | ENSG00000164220.6 | 71.457 KRT19P1 | ENSG00000218014.1 | 5.51 RP11-150012.3 ENSG00000254290.1 TMEM208 | ENSG00000168701.14 | 306 ASB13 | ENSG00000196372.8 | 306.0: GCSH | ENSG00000140905.5 | 187.771 PGK1 ENSG00000102144.9 9260.4

* HLA-DOA2 I ENSGO0000237541.3 I 14 IST1 | ENSG00000182149.16 | 1912.1 RP1-117B12.4 | ENSG00000253102.1 | PDRG1 | ENSG00000088356.4 | 224.57 PLOD2 | ENSG00000152952.7 | 2465.2 * IGKJ4 I ENSGO0000211594.2 | 168.34 * IGHG4 | ENSGO0000211892.2 | 414.42 * IGHV1-46 | ENSG00000211962.2 | 61 . * IGHV4-34 | ENSG00000211956.2 | 49 CTD-2554C21.3 | ENSG00000229481.1 * IGKV1-16 | ENSG00000240864.1 | 50. APRT | ENSGO0000198931.6 | 777.971 KARS | ENSG00000065427.10 | 2113.4 * IGHV4-39 | ENSG00000211959.2 | 52 s * IGHJ3 I ENSG00000242887.1 i 172.57 RP11-731F5.2 | ENSG00000253364.1 | * IGHV1-18 | ENSG00000211945.2 | 12: * IGrV4-31 | EASG00000231475.2 | 96. * IGHV4-55 i ENSG00000254395.1 18. CARID I ENSG00000213995.7 | $940.7 \%$ AL.139385.1 I ENSG00000255831.1 I s AK4P3 | ENSG00000233381.2 | 13.857 CFOMT1A | ENSG00000223572.5 | 75.26

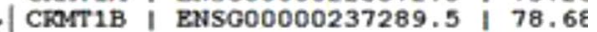

Figure 1. Genomic expression and characterization of eight resected brain metastases. Patients were divided between distant brain failure (DF) and no distant brain failure (NDF) for comparison. Significantly higher gene expression of immune cell populations were identified in the NDF group, as indicated by*.

Statistics. Patient outcomes were calculated from time of adjuvant SRS to event using competing risks estimates as previously described by McTyre $^{24}$. Brain metastasis velocity (BMV) was calculated for each patien as described by Farris et al. ${ }^{15}$. Kaplan-Meier estimates, and competing risk with subdistribution hazards and figures were calculated using version 3.4.0 software (R Foundation for Statistical Computing, Vienna, Austria). 


\begin{tabular}{|l|l|l|l|l|l|l|}
\hline Histology & $\begin{array}{l}\text { Number of BM treated at } \\
\text { adjuvant SRS }\end{array}$ & CD138 & $\begin{array}{l}\text { First IC } \\
\text { Event }\end{array}$ & $\begin{array}{l}\text { Months to 1st } \\
\text { IC event }\end{array}$ & BMV & $\begin{array}{l}\text { Overall survival } \\
\text { (months) }\end{array}$ \\
\hline Melanoma & 3 & 5 & DBF & 29 & 1.6 & 42 \\
\hline Melanoma & 2 & 7 & DBF & 8 & 26 & 15 \\
\hline SCLC & 1 & 22 & DBF & 2.5 & 26 & 12 \\
\hline Breast, Her2+ & 3 & 0 & DBF & 2 & 3.9 & 25 \\
\hline Breast, Her2- & 1 & 10 & NE & & 0 & 77 \\
\hline Breast, Her2- & 1 & 1 & LF+DBF & 3.2 & 0 & 22 \\
\hline NSCLC, adeno & 3 & 36 & LF+DBF & 7.3 & 3.5 & 11 \\
\hline NSCLC, adeno & 4 & 60 & DBF & 6.5 & 5.6 & 9 \\
\hline NSCLC, NOS & 5 & 1 & NE & & 0 & 4 \\
\hline Breast, Her2+ & 1 & 191 & NE & & 0 & 125 , alive \\
\hline NSCLC, adeno & 1 & 247 & NE & & 0 & 29 , alive \\
\hline NSCLC, NOS & 3 & 143 & NE & & 0 & 25 , alive \\
\hline NSCLC, NOS & 3 & 336 & DBF & 26 & 0.3 & 105 , alive \\
\hline
\end{tabular}

Table 1. Patient characteristics and CD138 expression. Brain Metastases (BM), Intracranial (IC), Brain metastasis velocity (BMV), Small cell lung cancer (SCLC), Non-small cell lung cancer (NSCLC), Adenocarcinoma (adeno), Not otherwise specified (NOS), Distant Brain Failure (DBF), Local Failure (LF), No event (NE).

\section{Results}

RNA sequencing and IHC. Eight patients with resected brain metastases were found to have several overexpressed B-cell related genes and a lower rate of DBF. Notably, the B-cell genes HLA-DQA2, IGKJ4, IGKV1-16, IGHG4, IGHJ3, IGHV1-18, IGHV1-24, IGHV1-46, IGHV4-28, IGHV4-31, IGHV4-34, IGHV4-39 and IGHV4-55, exhibited higher expression compared to patients who experienced a DBF at 58 months post-treatment (Fig. 1).

Thirteen patients were analyzed with IHC. This group of 13 patients had a median follow up of 29.5 months [Interquartile range (IQR) 11.1-37.5]. Histologies represented were melanoma $(\mathrm{N}=2)$, Breast $(\mathrm{N}=4)$, non-small cell lung cancer $(\mathrm{N}=6)$, and small cell lung cancer $(\mathrm{N}=1)$ (Table 1$)$. The patient with small cell lung cancer had received prophylactic cranial irradiation 2 years prior to craniotomy, no other patients received whole brain radiation prior to craniotomy. Twelve of the 13 patients received steroids prior to craniotomy for brain metastasis; it remained unknown if the remaining patient received steroids. The median KPS was 90 [IQR 70-90]. At time of analysis, 2 local failures had occurred, 8 distant brain failures were observed, 4 patients experienced leptomeningeal failure, 4 patients received salvage WBRT, and 9 patients had died.

PD-L1 was highly expressed in three patients and PD1 overexpressed in 1 patient. High expression of CD3 was observed in 9 patients and high expression of CD20 was observed in 4 patients. CD138 was quantitatively assessed and demonstrated bimodal distribution pattern. Nine patients had CD138 cell counts of 60 or less (range $0-60$ ) and 4 patients had CD138 cell counts of greater than 140 (range 143-247). Figure 2 is a representative picture of $\mathrm{H} \& \mathrm{E}$ stains.

Of the patients with high expression of CD138, no deaths have been observed, no local failures, and one DBF has been recorded. All patients with high expression of CD138 were still alive. Of these patients, 3 had NSCLC and 1 had breast cancer. The median OS in patients with low expression of CD138 was 14.7 months vs not reached for high expression of CD138 (Log Rank $\mathrm{p}=0.01)$ (Fig. 3). Competing risk for time to first intracranial event between $\mathrm{CD} 138$ high expression vs low expression did not reach statistical significance $(\mathrm{P}=0.11)$. Univariate analysis of other IHC stains (CD3, CD20, PD-1, PDL1) did not reveal a statistically significant association between cell marker expression and OS or intracranial failure.

\section{Discussion}

For patients who underwent craniotomy followed by adjuvant SRS for brain metastases, high levels of CD138 lymphocytes within craniotomy samples were associated with improved survival compared to patients with low levels of CD138 lymphocytes. CD138 is a plasma cell marker and a proteoglycan, syndecan 1, which is expressed by solid tumor cells and plasma cells. When expressed by tumor cells, CD138 has been associated with metastasis and poor survival ${ }^{25,26}$. However, the infiltration of CD138 plasma cells in solid tumors has been associated with longer survival in patients with ovarian, gastroesophageal, NSCLC, and colon cancers ${ }^{27}$. These effects might be augmented by the localization within tumor, which would enable high concentrations of antibody to accumulate locally. Antibodies could opsonize tumor antigens, thereby facilitating antigen presentation and broadening of $\mathrm{T}$-cell responses ${ }^{28-30}$. Moreover, antibodies could mediate direct antitumor effects by binding to and disrupting the function of their cognate antigens, activating the complement pathway, and/or triggering antibody-dependent cellular cytotoxicity ${ }^{31}$.

Unlike biomarkers that predict for brain metastasis-specific response to a systemic agent, a biomarker with the capability of predicting SRS outcomes in patients with brain metastases from different primary tumors would have significant clinical potential given the current dilemma by which patients are selected for upfront SRS versus upfront WBRT. Several statistical models have been developed in order to help triage patients, but the ability of validate them has been somewhat questionable ${ }^{14,32}$. A major issue with validation of predictive models for brain metastases has been the biological heterogeneity of brain metastases and the fact that brain metastases of different primary tumors have distinct natural histories due to variations in systemic disease burden and control ${ }^{5,33}$. The 


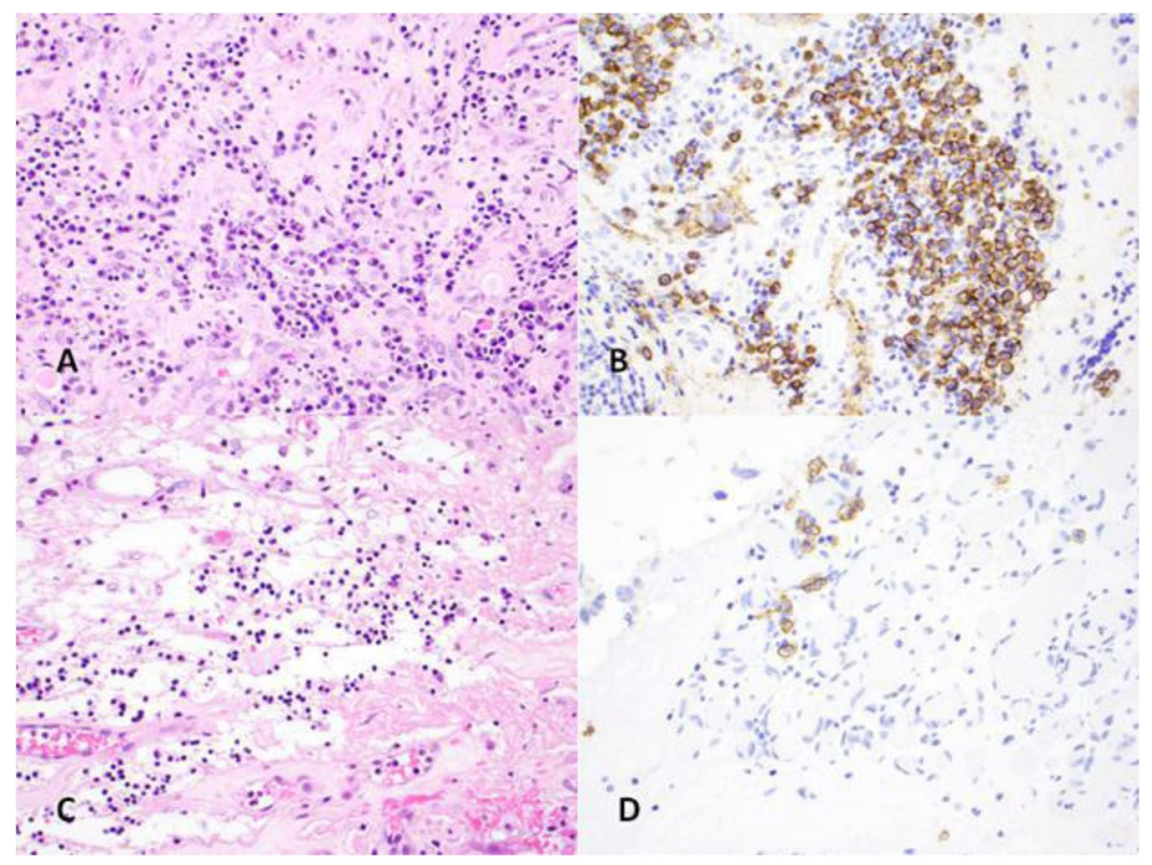

Figure 2. (A) and (B), a case with high plasma cells. (C,D) A case with low plasma cells. [(A) and (C), hematoxylin and eosin. (B) and (D), CD138 immunohistochemical stain. All images taken at 40X magnification].

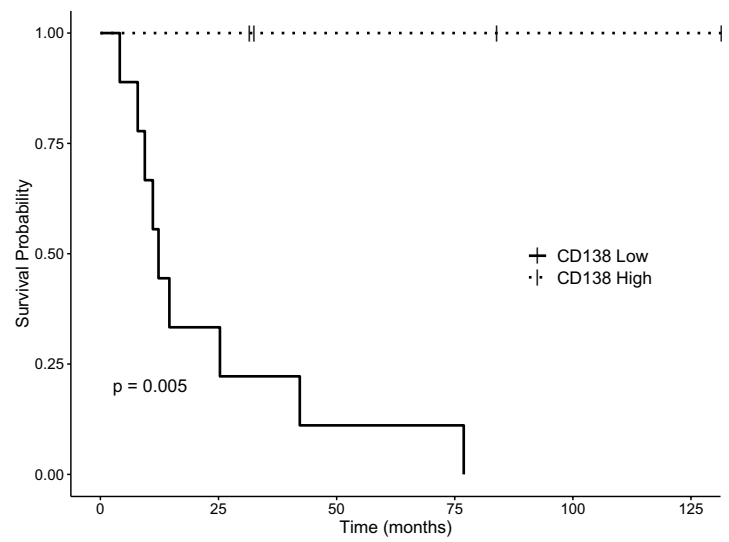

Figure 3. Kaplan Meier estimates of overall survival for patients with stratified by high or low CD138 positivity.

discovery of the immunotype changes that drive these biological differences will hopefully help to improve the predictability of brain metastasis outcomes moving forward.

This study is limited by a small sample size, retrospective nature, and requires further validation in a large cohort of patients prior to utilizing CD138 as a biomarker for clinical practice. However, the identification of an immune marker within resected brain metastasis tissue that translates into improved survival outcomes is an exciting finding and warrants further investigation.

\section{Conclusion}

Patients with high levels of CD138 expressing plasma cells may have improved OS compared to patients with low levels of CD138 with a trend towards fewer intracranial failures. The results are hypothesis generating and CD138 expression should be investigated in a larger cohort of patients with resected brain metastasis tissue.

\section{References}

1. Ellis, T. L., Neal, M. T. \& Chan, M. D. The role of surgery, radiosurgery and whole brain radiation therapy in the management of patients with metastatic brain tumors. Int. J. Surg. Oncol. 2012, 952345 (2012).

2. Ayala-Peacock, D. N. et al. A nomogram for predicting distant brain failure in patients treated with gamma knife stereotactic radiosurgery without whole brain radiotherapy. Neuro. Oncol. 16, 1283-1288 (2014).

3. Kuremsky, J. G. et al. Tumor histology predicts patterns of failure and survival in patients with brain metastases from lung cancer treated with gamma knife radiosurgery. Neurosurgery 73, 641-7; discussion 647 (2013). 
4. Harris, S. et al. Gamma knife stereotactic radiosurgery as salvage therapy after failure of whole-brain radiotherapy in patients with small-cell lung cancer. Int. J. Radiat. Oncol. Biol. Phys. 83, e53-9 (2012).

5. Lucas, J. T. Jr. et al. Competing Risk Analysis of Neurologic versus Nonneurologic Death in Patients Undergoing Radiosurgical Salvage After Whole-Brain Radiation Therapy Failure: Who Actually Dies of Their Brain Metastases? Int. J. Radiat. Oncol. Biol. Phys. 92, 1008-1015 (2015).

6. Savitz, S. T., Chen, R. C. \& Sher, D. J. Cost-effectiveness analysis of neurocognitive-sparing treatments for brain metastases. Cancer 121, 4231-4239 (2015).

7. Aoyama, H. et al. Stereotactic radiosurgery plus whole-brain radiation therapy vs stereotactic radiosurgery alone for treatment of brain metastases: a randomized controlled trial. JAMA 295, 2483-2491 (2006).

8. Chang, E. L. et al. A pilot study of neurocognitive function in patients with one to three new brain metastases initially treated with stereotactic radiosurgery alone. Neurosurgery 60, 277-83; discussion 283-4 (2007).

9. Soffietti, R. et al. A European Organisation for Research and Treatment of Cancer phase III trial of adjuvant whole-brain radiotherapy versus observation in patients with one to three brain metastases from solid tumors after surgical resection or radiosurgery: quality-of-life results. J. Clin. Oncol. 31, 65-72 (2013).

10. Brown, P. D. et al. NCCTG N0574 (Alliance): A phase III randomized trial of whole brain radiation therapy (WBRT) in addition to radiosurgery (SRS) in patients with 1 to 3 brain metastases. J. Clin. Orthod. 33, LBA4-LBA4 (2015).

11. Thomas, E. M. et al. Comparison of plan quality and delivery time between volumetric arc therapy (RapidArc) and Gamma Knife radiosurgery for multiple cranial metastases. Neurosurgery 75, 409-17; discussion 417-8 (2014).

12. Lester, S. C. et al. Clinical and economic outcomes of patients with brain metastases based on symptoms: an argument for routine brain screening of those treated with upfront radiosurgery. Cancer 120, 433-441 (2014).

13. Press, R. H. et al. Novel risk stratification score for predicting early distant brain failure and salvage whole-brain radiotherapy after stereotactic radiosurgery for brain metastases. Cancer 121, 3836-3843 (2015).

14. Ayala-Peacock, D. N. et al. Prediction of new brain metastases after radiosurgery: validation and analysis of performance of a multiinstitutional nomogram. J. Neurooncol., https://doi.org/10.1007/s11060-017-2588-4 (2017).

15. Farris, M. et al. Brain Metastasis Velocity: A Novel Prognostic Metric Predictive of Overall Survival and Freedom From Whole-Brain Radiation Therapy After Distant Brain Failure Following Upfront Radiosurgery Alone. Int. J. Radiat. Oncol. Biol. Phys. 98, 131-141 (2017).

16. Church, S. E. \& Galon, J. Tumor Microenvironment and Immunotherapy: The Whole Picture Is Better Than a Glimpse. Immunity 43, 631-633 (2015).

17. Hamilton, A. \& Sibson, N. R. Role of the systemic immune system in brain metastasis. Mol. Cell. Neurosci. 53, 42-51 (2013).

18. Berghoff, A. S. et al. Density of tumor-infiltrating lymphocytes correlates with extent of brain edema and overall survival time in patients with brain metastases. Oncoimmunology 5, e1057388 (2016).

19. Shaw, E. et al. Single dose radiosurgical treatment of recurrent previously irradiated primary brain tumors and brain metastases: final report of RTOG protocol 90-05. Int. J. Radiat. Oncol. Biol. Phys. 47, 291-298 (2000).

20. Jensen, C. A. et al. Cavity-directed radiosurgery as adjuvant therapy after resection of a brain metastasis. J. Neurosurg. 114, 1585-1591 (2011).

21. Dobin, A. et al. STAR: ultrafast universal RNA-seq aligner. Bioinformatics 29, 15-21 (2013).

22. Liao, Y., Smyth, G. K. \& Shi, W. featureCounts: an efficient general purpose program for assigning sequence reads to genomic features. Bioinformatics 30, 923-930 (2014).

23. Love, M. I., Huber, W. \& Anders, S. Moderated estimation of fold change and dispersion for RNA-seq data with DESeq. 2. Genome Biol. 15, 550 (2014).

24. McTyre, E. R. et al. Predictors of neurologic and nonneurologic death in patients with brain metastasis initially treated with upfront stereotactic radiosurgery without whole-brain radiation therapy. Neuro. Oncol., https://doi.org/10.1093/neuonc/now184 (2016)

25. Zhou, J. et al. Surface profiles of live colorectal cancer cells and tumor infiltrating lymphocytes from surgical samples correspond to prognostic categories. J. Immunol. Methods 416, 59-68 (2015).

26. Luque-García, J. L. et al. Differential protein expression on the cell surface of colorectal cancer cells associated to tumor metastasis. Proteomics 10, 940-952 (2010).

27. Lohr, M. et al. The prognostic relevance of tumour-infiltrating plasma cells and immunoglobulin kappa C indicates an important role of the humoral immune response in non-small cell lung cancer. Cancer Lett. 333, 222-228 (2013).

28. Brahmer, J. R. Harnessing the immune system for the treatment of non-small-cell lung cancer. J. Clin. Oncol. 31, 1021-1028 (2013).

29. Baggers, J., Ratzinger, G. \& Young, J. W. Dendritic cells as immunologic adjuvants for the treatment of cancer. J. Clin. Oncol. 18, 3879-3882 (2000).

30. Inogés, S. et al. A phase II trial of autologous dendritic cell vaccination and radiochemotherapy following fluorescence-guided surgery in newly diagnosed glioblastoma patients. J. Transl. Med. 15, 104 (2017).

31. Vidarsson, G., Dekkers, G. \& Rispens, T. IgG subclasses and allotypes: from structure to effector functions. Front. Immunol. 5, 520 (2014).

32. Gorovets, D. et al. Multi-institutional Nomogram Predicting Survival Free From Salvage Whole Brain Radiation After Radiosurgery in Patients With Brain Metastases. Int. J. Radiat. Oncol. Biol. Phys. 97, 246-253 (2017).

33. Johnson, A. G. et al. Impact of systemic targeted agents on the clinical outcomes of patients with brain metastases. Oncotarget 6, 18945-18955 (2015).

\section{Acknowledgements}

The work is supported by the Cancer Center Support Grant from the National Cancer Institute to the Comprehensive Cancer Center of Wake Forest Baptist Medical Center (P30 CA012197) and Brain Tumor Center of Excellence.

\section{Author Contributions}

Michael H. Soike, M.D. prepared and submitted the manuscript. Jennifer Logue, M.D. identified the patients, performed analysis, and contributed to the manuscript. Shadi Qasem, M.D. provided pathology support for this work. Ryan T. Hughes, M.D. provided statistical support and edited the manuscript. Emory McTyre, M.D. provided statistical support and edited the manuscript. Jing Su, Ph.D. provided statistical support and performed the genomic analysis. Pierre Triozzi, M.D. added perspective from the immunotherapy standpoint. Maurizio Bendandi, MD, Ph.D significantly contributed to the manuscript. Hui-Wen Lo, Ph.D. contributed to the discussion. Tamjeed Ahmed, M.D. contributed to the edits for the discussion. Stacey S. O'Neill, M.D., Ph.D. provided second pathology validation and verification of the samples. Waldemar Debinski, Ph.D. significantly edited and revised the manuscript. Boris Pasche, M.D., Ph.D. significantly edited and revised the manuscript. Kounosuke Watabe, Ph.D. significantly edited and revised the manuscript and helped direct the basic science 
component. Lance D. Miller, Ph.D. created the Figure 3. Michael D. Chan, M.D. wrote the IRB, contributed to the text. Jimmy Ruiz, M.D. acted as senior author and adviser and made substantial contributions to the introduction and the discussion.

\section{Additional Information}

Competing Interests: The authors declare no competing interests.

Publisher's note Springer Nature remains neutral with regard to jurisdictional claims in published maps and institutional affiliations.

(c) (i) Open Access This article is licensed under a Creative Commons Attribution 4.0 International License, which permits use, sharing, adaptation, distribution and reproduction in any medium or format, as long as you give appropriate credit to the original author(s) and the source, provide a link to the Creative Commons license, and indicate if changes were made. The images or other third party material in this article are included in the article's Creative Commons license, unless indicated otherwise in a credit line to the material. If material is not included in the article's Creative Commons license and your intended use is not permitted by statutory regulation or exceeds the permitted use, you will need to obtain permission directly from the copyright holder. To view a copy of this license, visit http://creativecommons.org/licenses/by/4.0/.

(C) The Author(s) 2019 